\title{
Calcium neuroimaging in behaving zebrafish larvae using a turn-key light field camera
}

Carlos Cruz Perez

Antonella Lauri

Panagiotis Symvoulidis

Michele Cappetta

Arne Erdmann

Gil Gregor Westmeyer 


\title{
Calcium neuroimaging in behaving zebrafish larvae using a turn-key light field camera
}

\author{
Carlos Cruz Perez, ${ }^{\mathrm{a}, \mathrm{b}, \dagger}$ Antonella Lauri, ${ }^{\mathrm{a}, \mathrm{b}, \mathrm{c}, \dagger}$ Panagiotis Symvoulidis, ${ }^{\mathrm{a}, \mathrm{b}, \mathrm{c}, \dagger}$ Michele Cappetta, ${ }^{\mathrm{a}, \mathrm{b}, \mathrm{c}}$ \\ Arne Erdmann, ${ }^{\mathrm{d}}$ and Gil Gregor Westmeyer ${ }^{\mathrm{a}, \mathrm{b}, \mathrm{c}, \mathrm{e}, \mathrm{e}, \mathrm{t}}$ \\ ${ }^{a}$ Helmholtz Zentrum München, Institute of Biological and Medical Imaging, Ingolstädter Landstrasse 1, 85764 Neuherberg, Germany

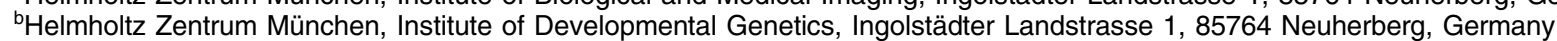 \\ 'Technische Universität München, Department of Nuclear Medicine, Ismaningerstrasse 22, 81675 Munich, Germany \\ ${ }^{\mathrm{d}}$ Raytrix, Schauenburgerstrasse 116, 24118 Kiel, Germany

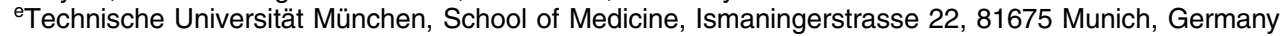

\begin{abstract}
Reconstructing a three-dimensional scene from multiple simultaneously acquired perspectives (the light field) is an elegant scanless imaging concept that can exceed the temporal resolution of currently available scanning-based imaging methods for capturing fast cellular processes. We tested the performance of commercially available light field cameras on a fluorescent microscopy setup for monitoring calcium activity in the brain of awake and behaving reporter zebrafish larvae. The plenoptic imaging system could volumetrically resolve diverse neuronal response profiles throughout the zebrafish brain upon stimulation with an aversive odorant. Behavioral responses of the reporter fish could be captured simultaneously together with depth-resolved neuronal activity. Overall, our assessment showed that with some optimizations for fluorescence microscopy applications, commercial light field cameras have the potential of becoming an attractive alternative to custom-built systems to accelerate molecular imaging research on cellular dynamics. $\odot$ The Authors. Published by SPIE under a Creative Commons Attribution 3.0 Unported License. Distribution or reproduction of this work in whole or in part requires full attribution of the original publication, including its DOI. [DOI: 10.1117/1.JBO.20.9.096009]
\end{abstract}

Keywords: light field microscopy; plenoptic microscopy; calcium imaging; zebrafish neuroimaging; behavior.

Paper 150224R received Apr. 3, 2015; accepted for publication Aug. 4, 2015; published online Sep. 10, 2015.

\section{Introduction}

Neuronal activity occurs on a millisecond time scale across cell circuits distributed over the entire nervous system. Capturing these spatiotemporal patterns at adequate sampling rates to uncover the underlying principles of neuronal information processing is a grand technical challenge for systems neuroscience. Recently, it became possible in transparent reporter zebrafish larvae expressing genetically encoded fluorescent calcium indicators (GECIs) ${ }^{1,2}$ to detect calcium transients noninvasively in the whole nervous system. In this vertebrate model organism, imaging can thus deliver information about a close correlate of neuronal activation, calcium fluxes, at much higher spatial resolution than is achievable with electrophysiological point recordings. To exceed the speed of confocal or multiphoton laser scanning methods, ${ }^{3}$ light sheet microscopy methods have recently been developed ${ }^{4-6}$ that image an entire plane through the object of interest at once, such that about $\sim 10^{5}$ neurons in an entire zebrafish larval brain can be scanned at a frequency of about $1 \mathrm{~Hz}{ }^{4}$ Volumetric acquisition can be accelerated even further by light field imaging, an elegant method that avoids having to scan the object but instead reconstructs it volumetrically from multiple views acquired simultaneously from many different angles. This imaging method has recently been adapted for microscopy by using arrays of microlenses projecting to

\footnotetext{
*Address all correspondence to: Gil Gregor Westmeyer, E-mail: gil.westmeyer@ tum.de

${ }^{\dagger}$ Authors contributed equally.
}

subregions of sufficiently sensitive and large image sensors. ${ }^{7,8}$ Light field microscopy (also known as plenoptic microscopy) could thus, in principle, capture the majority of fluorescent neurons in the brain of a reporter zebrafish in a single acquisition without the need for interpolation to correct for time delays. Neuroimaging of reporter zebrafish would benefit directly from such a gain in imaging speed. For instance, state-of-the-art fast light sheet microscopy, operating at about one volume per second, still misses a substantial fraction of calcium fluctuations detected by sensors such as GCaMP5G (rise times of $\sim 0.2 \mathrm{~s}$ and decay rates of $\left.\sim 0.7 \mathrm{~s}^{1,4}\right)$. For faster fluorescent sensors, such as genetically encoded voltage sensors, ${ }^{9}$ even higher frame rates ${ }^{10}$ would be required for adequate sampling. Furthermore, fast volumetric imaging could simultaneously extract information from fluorescent molecular sensors together with "biomechanical" data from freely moving zebrafish larvae exhibiting unrestrained behavior; this may complement virtual environment approaches that investigate neural activity during fictive behavior in immobilized zebrafish larvae. ${ }^{11}$ Lately, technical advances have been made in acquisition and reconstruction of light field microscopy data ${ }^{12-14}$ and three light field microscopes have been custom-built in different laboratories specifically to image neuronal activity in immobilized Caenorhabditis elegans worms and zebrafish larvae. ${ }^{8,14,15}$ Despite the generous provision of open access documentation on light field microscopy hardware and open source software, ${ }^{16,17}$ an "off-the shelf" light field camera system would certainly help to disseminate this comparably straightforward and compact imaging technology for widespread biological use. In this work, we thus investigated the performance of commercially available multifocus plenoptic 
cameras $^{18}$ (Raytrix $\mathrm{GmbH}$ ) for fluorescence neuroimaging of zebrafish larvae.

\section{Results and Discussion}

For initial testing of the commercial light field camera for microscopy, we imaged the distribution of melanin containing pigments in the head region of wild-type zebrafish larvae (6 days post fertilization) using a custom-assembled microscopy setup [Fig. 1(a)]. The larvae were embedded in $0.8 \%$ low-melting agarose and imaged under white light illumination $(10 \times$ objective from Nikon, $0.25 \mathrm{NA}$, FOV of $\sim 1.9 \mathrm{~mm} \times 1.3 \mathrm{~mm}, \mathrm{R} 9 \mu$ plenoptic camera; all animal experiments were conducted in accordance with the guidelines approved by the government of Upper Bavaria). As can be seen in Fig. 1, the head melanophores of the larva and the eyes offered high contrast [Fig. 1(b)] and their localization along the dorsoventral axis was correctly captured by the depth map calculated from the light field images exploiting information from microlenses with different focal lengths [Fig. 1(c)]. We subsequently tested the performance of the plenoptic camera for fluorescence microscopy by imaging green fluorescent beads of $1 \mu \mathrm{m}$ in diameter (FluoSpheres carboxylate, Life Technologies, Carlsbad, California) embedded in an agar phantom. The sample excitation and fluorescence detection was achieved via a 488-nm OBIS laser (Coherent), a dichroic mirror (510 nm, Chroma Technology Corp.), a 20× objective (UMPlanFl N 20×, $0.5 \mathrm{NA}$, FOV $650 \mu \mathrm{m} \times 440 \mathrm{~mm}$ ) a 535/40 nm emission filter (Chroma), and a R12 $\mu$ plenoptic camera. Figure 1(d) shows the projection of single microspheres onto the image sensor, obtained via the microlens array
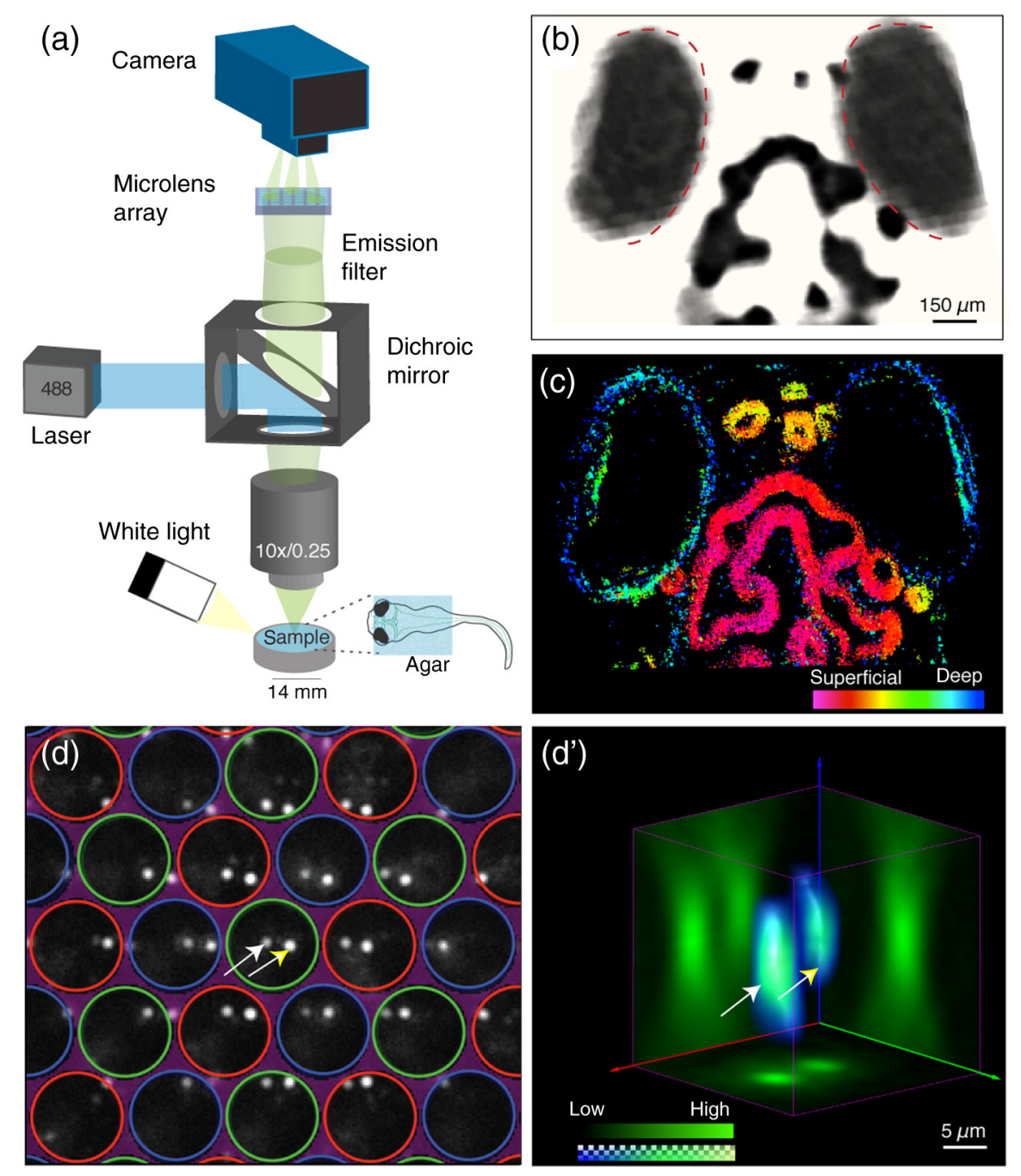

Fig. 1 Light field microscopy setup and volumetric imaging in bright field and fluorescence: (a) Fluorescent light from the specimen is guided via a dichroic mirror and emission filter to a microlens array projecting it to different subregions of the plenoptic camera's image sensor (reflectance images from white light illumination are captured without filters). (b) Reconstructed light field data obtained from a wild-type larva showing strong contrast from naturally present pigments in the eyes (red dashed outlines) and in the head region; (c) Corresponding depth information calculated from the light field data with most superficial structures shown in magenta and deeper structures in blue; (d) Raw grayscale image showing two fluorescence beads ( $1 \mu \mathrm{m}$ in diameter, white and yellow arrows) embedded in an agar phantom projected onto the image sensor via microlenses with different focal lengths (red/ green/blue circles label microlenses with close/medium/far focal lengths); and (d') Three-dimensional visualization of the same two volumetrically reconstructed fluorescent spheres (white and yellow arrows). 

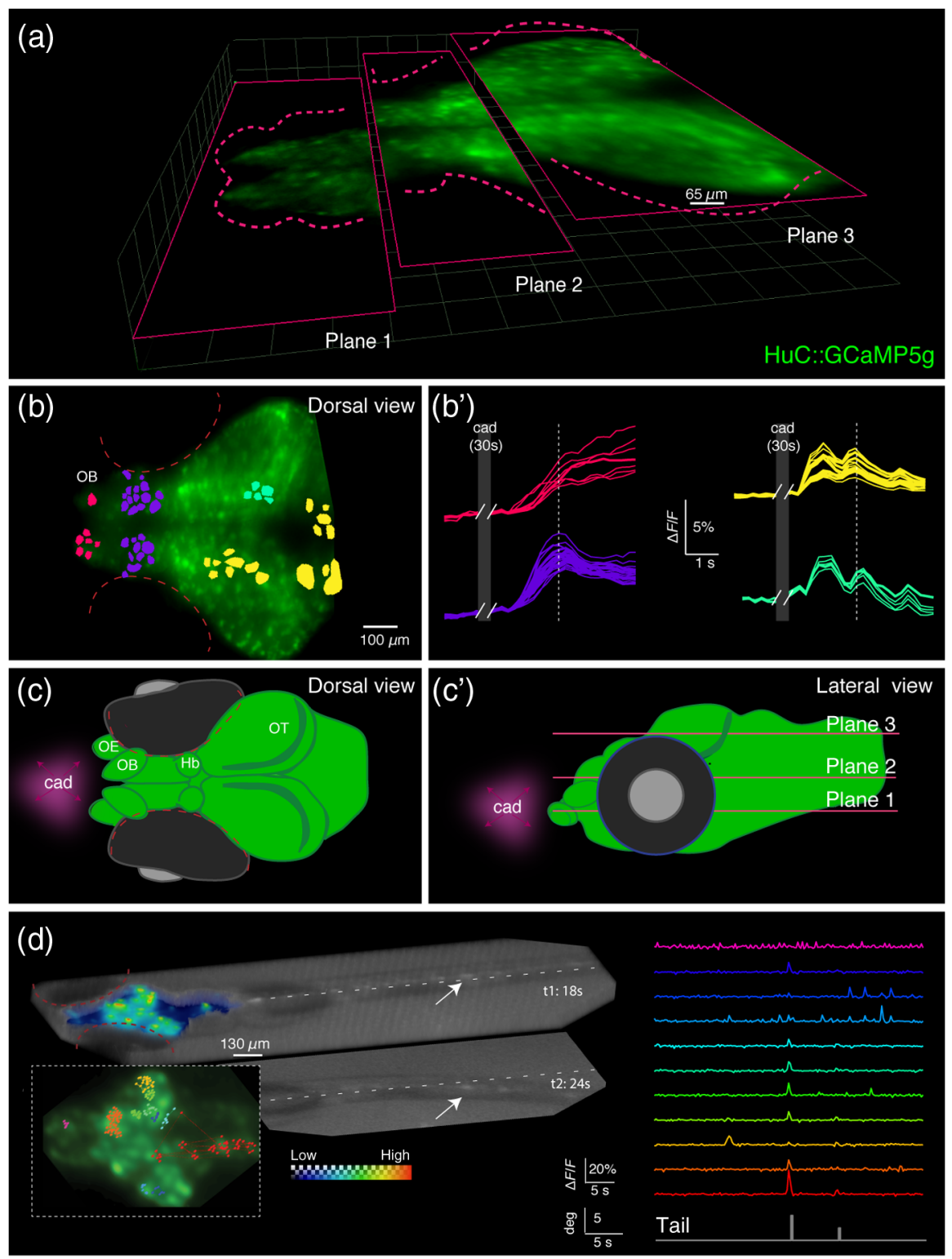

Fig. 2 Spatiotemporal patterns of neuronal responses to an aversive odor captured by light field microscopy: (a) Three transverse planes selected from the volumetric reconstruction of a single frame captured by the plenoptic camera showing fluorescence signals from the brain of a calcium reporter zebrafish larva (7-day-old HuC:GCaMP5g) during stimulation with the aversive odorant cadaverine; (b-b') Corresponding top-down maximum intensity projection with a colored overlay of regions of interest (ROIs) automatically selected for their substantial fluorescent signal changes in response to cadaverine. The color indicates the clusters of ROIs with high correlation between their corresponding temporal signal profiles shown in (b'). Delivery of cadaverine occurred during the omitted time points (dark gray area labeled "cad"). The white broken lines indicate the peak fluorescence signal of the deep purple trace as a reference to appreciate the earlier responses in the yellow and green traces; (c-c') Schematic drawings of the larval brain seen from dorsal (c) and lateral views (c') also indicating the site where the odorant cadaverine (cad) was presented. OE: olfactory epithelium, OB: olfactory bulb, OT: optic tectum, and $\mathrm{Hb}$ : habenula. (d) Rendered imaging volume showing simultaneous imaging of neuronal activity (high counts from fluorescence shown on color scale) and tail movements (low counts shown on grayscale) in response to cadaverine stimulation [deflection of the tail's axis from the dashed white line is shown for a second time point (t2)]. Clustered ROls are shown on a top-down maximum intensity projection in the inset on the lower left and color-coded signal time courses are shown on the right. Tail deflections coincident with some neuronal activations are represented as gray bars.

consisting of microlenses with different focal lengths assembled in a hexagonal configuration. ${ }^{18}$ The corresponding volumetric reconstruction is shown in Fig. 1(d') yielding a lateral resolution of $6 \mu \mathrm{m}$ and an axial resolution of $16 \mu \mathrm{m}$ (estimated by the averaged point spread function without additional postprocessing).
We were next interested to assess whether the light field microscopy setup could detect dynamic changes of neuronal activity in transgenic reporter zebrafish larvae with neuronal expression of a calcium indicator (HuC:GCaMP5g, identical optical setup as described above). To this end, we restrained 
zebrafish larvae in $0.8 \%$ low-melting agarose not covering the nose. We stimulated the larvae with the odorant cadaverine (Cad, 10- $\mu \mathrm{M}$ solution), a diamine generated by decarboxylation of the amino acid lysine during decomposition of flesh, which is known to evoke olfactory-related neural activity and avoidance responses in zebrafish larvae. ${ }^{19,20}$ Figure 2 shows three image planes through the volumetrically reconstructed light field dataset [Fig. 2(a)], as well as a top-down maximum intensity projection [Fig. 2(b)] showing robust fluorescent signal. Regions of interest (ROIs) could be automatically selected based on thresholding voxel signal changes in response to cadaverine and clustered with respect to the correlation (correlation coefficient larger than 0.8 ) of their respective baselinecorrected time courses [color coded in Fig. 2(b')]. Clusters of activity could be found in the olfactory bulb (OB) and habenula $(\mathrm{Hb})$, the latter considered to be involved in relaying odorevoked behavioral responses, ${ }^{19,20}$ as well as in some neurons of the optic tectum (OT) [Figs. 2(b)-2(c)]. Whereas calcium signal amplitudes increased in the $\mathrm{OB}$ after cadaverine delivery [the delivery period is labeled "cad" on the gray area in Fig. 2(b)] during the observed time window, they partially decreased after peaking a few seconds after delivery of cadaverine (white broken lines in all traces); signal trajectories in the OT showed the first response peak occurring $\backsim 1 \mathrm{~s}$ earlier than in the OB followed by multiple weaker calcium waves.

Next, we explored whether we could simultaneously capture neuronal activity while recording behavioral responses to the aversive odorant using the light field microscopy setup. To this aim, we embedded HuC:GCaMP5g larvae in agarose such that the tail was free to move and chose a $5 \times$ objective to obtain a field of view covering the entire larva. After cadaverine delivery, evoked neuronal calcium fluxes were recorded while concurrent tail movements were detected that are indicative of avoidance responses ${ }^{21}$ [Fig. 2(d)]. Cluster analysis on automatically detected ROIs on this dataset interestingly showed activation in areas of the hindbrain that were in synchrony with the tail movements [gray bars underneath the signal time courses in Fig. 2(d)].

In summary, using a commercially available plenoptic camera (Raytrix $\mathrm{GmbH}$ ) mounted on a custom-built microscope, we were able to volumetrically resolve calcium-dependent fluorescent signal changes elicited by the aversive odorant cadaverine in the brains of behaving zebrafish larvae. The capability of light field microscopy to acquire an entire volume in a single shot enabled us to detect diverse temporal response profiles from neuronal structures at different depths throughout the larval brain that motivate future detailed investigations. The current turn-key plenoptic camera could be successfully used to extract meaningful volumetric information about calcium signaling in distinct brain regions of reporter fish. However, both the spatial and temporal resolutions of the imaging system could be strongly improved by combining custom-designed multilens arrays and optimized reconstruction algorithms with scientific sCMOS image sensors that should provide an about four times higher sensitivity. These hardware improvements could be effectively complemented by the use of reporter fish expressing GECIs localized to the cell nucleus (as opposed to the entire cell), thereby aiding volumetric extraction of cellular calcium transients from the nonisotropic voxels obtained from current light field microscopy setups. As light field imaging is an elegant solution for fast molecular bioimaging applications, it is to be hoped that plenoptic cameras could be further optimized for fast fluorescence microscopy and be made more widely available to the biomedical research community.

\section{Acknowledgments}

Shared authorships are listed in alphabetical order: A.L., P.S., A. E., C.C.-P., and G.G.W. conducted the experiments; M.C., P.S., A.L., A.E., and G.G.W. analyzed the data; and A.L., P.S., and G. G.W. wrote the manuscript. We thank Raytrix GmbH (Kiel, Germany) for their assistance with the light field camera during our experiments, Dr. Michael Orger (Champalimaud Centre for the Unknown, Lisbon, Portugal) and Dr. Hernan Lopez-Schier (SBO, Helmholtz Zentrum Muenchen, Germany) for sharing the zebrafish transgenic line HuC:GCaMP5g. G.G.W. and C.C.-P. acknowledge the support from the Helmholtz Alliance ICEMED. A.L. acknowledges the support from the Postodoctoral Fellowship Program (PFP) of the Helmholtz Zentrum München. G.G.W. and A.L. acknowledge the support from the European Research Council under grant agreement ERC2012-StG- 311552.

\section{References}

1. J. Akerboom et al., "Optimization of a GCaMP calcium indicator for neural activity imaging," J. Neurosci. 32(40), 13819-13840 (2012).

2. J. Akerboom et al., "Genetically encoded calcium indicators for multicolor neural activity imaging and combination with optogenetics," Front. Mol. Neurosci. 6, 2 (2013).

3. D. Dombeck and D. Tank, "Two-photon imaging of neural activity in awake mobile mice," Cold Spring Harbor Protoc. 2014(7), 726-736 (2014).

4. M. B. Ahrens et al., "Whole-brain functional imaging at cellular resolution using light-sheet microscopy," Nat. Methods 10(5), 413-420 (2013).

5. P. J. Keller et al., "Reconstruction of zebrafish early light sheet microscopy," Science 322(5904), 1065-1069 (2008).

6. F. O. Fahrbach et al., "Rapid 3D light-sheet microscopy with a tunable lens," Opt. Express 21(18), 21010-21026 (2013).

7. M. Levoy and P. Hanrahan, "Light field rendering," in Proc. 23rd Annual Conf. Computer Graphics and Interactive TechniquesSIGGRAPH, Association for Computing Machinery (ACM) (1996).

8. R. Prevedel et al., "Simultaneous whole-animal 3D imaging of neuronal activity using light-field microscopy," Nat. Med. 11, 727-732 (2014).

9. Y. Gong, J. Z. Li, and M. J. Schnitzer, "Enhanced archaerhodopsin fluorescent protein voltage indicators," PLoS One 8(6), e66959 (2013).

10. F. St-Pierre et al., "High-fidelity optical reporting of neuronal electrical activity with an ultrafast fluorescent voltage sensor," Nat. Neurosci. 17(6), 884-889 (2014).

11. N. Vladimirov et al., "Light-sheet functional imaging in fictively behaving zebrafish," Nat. Methods 11(9), 883-884 (2014).

12. N. Cohen et al., "Enhancing the performance of the light field microscope using wavefront coding," Opt. Express 22(20), 24817-24839 (2014).

13. M. Broxton et al., "Wave optics theory and 3-D deconvolution for the light field microscope," Opt. Express 21(21), 25418-25439 (2013).

14. I. Iglesias and J. Ripoll, "Plenoptic projection fluorescence tomography," Opt. Express 22(19), 23215-23225 (2014).

15. L. Grosenick et al., "Elastic source selection for in vivo imaging of neuronal ensembles," in 2009 IEEE International Symposium on Biomedical Imaging: From Nano to Macro, pp. 1263-1266 (2009).

16. A. Vaziri et al., "Lightfield microscopy for high-speed, three-dimensional, simultaneous imaging of neural dynamics," in Simultaneous whole-animal $3 D$ imaging of neuronal activity using light-field microscopy, Prevedel et al., Eds., Nature Methods 11, pp. 727-730, http:// www.lightfieldscope.org/ (2014).

17. M. Levoy et al., "Stanford Light Field Microscope Project," http:// graphics.stanford.edu/projects/lfmicroscope/ (8 2015).

18. C. Perwaß, L. Wietzke, and R. Gmbh, "Single lens 3D-camera with extended depth-of-field" Human Vision and Electronic Imaging XVII, Vol. 8291 (2012). 
19. A. Hussain et al., "High-affinity olfactory receptor for the deathassociated odor cadaverine," Proc. Natl. Acad. Sci. U. S. A. 110(48), 19579-19584 (2013).

20. S. Krishnan et al., "The right dorsal habenula limits attraction to an odor in zebrafish," Curr. Biol. 24(11), 1167-1175 (2014).

21. A. V. Kalueff et al., "Towards a comprehensive catalog of zebrafish," Zebrafish 10(1), 70-86 (2013).

Carlos Cruz Pérez received his Diploma in Telecommunication Engineering from Technical University of Madrid in 2013. He then joined the Institutes of Biological and Medical Imaging (IBMI) and Developmental Genetics (IDG) at Helmholtz Zentrum München to work on optical systems for neuroimaging.

Antonella Lauri received her MS degree in medical biotechnology from the University of Naples "Federico II," Italy, in 2008. In 2013, she obtained her $\mathrm{PhD}$ (and Doctor of Science) from Heidelberg University and EMBL (European Molecular Biology Laboratory, Germany), applying different molecular and imaging methods to solve evolutionary and developmental questions. In 2013, she joined the Institutes of Biological and Medical Imaging and Developmental Genetics at Helmholtz Zentrum Muenchen, where she focuses on fish neuroimaging.

Panagiotis Symvoulidis received his Diploma in electrical and computer engineering from Technical University of Athens (NTUA), where he focused his studies on signal processing and electronics for biomedical engineering application. Subsequently, he moved to the Technische Universitaet Muenchen, where he worked toward his $\mathrm{PhD}$ under the supervision of Prof. V. Ntziachristos on the systems and algorithms for fluorescence and multispectral tissue imaging in the diffusive regime. Recently, he joined Prof. Westmeyer's laboratory at Helmholtz Zentrum Muenchen, developing equipment and methods for neuroimaging studies.

Michele Cappetta has studied at the "University of the studies of Trieste" (Italy) where he obtained a Bachelor in physics in 2006 and his MS degree in astrophysics in 2008. In 2013 he concluded his $\mathrm{PhD}$ in physics at the Ludwig Maximilian University Munich conducting a research project on extrasolar planets at the Max-PlanckInstitute for Extraterrestrial Physics in Munich. He became a member of IBMI and IDG the Helmholtz Zentrum Muenchen in 2014.

Gil Gregor Westmeyer completed his dissertation in medicine from Ludwig Maximilian University Munich. He conducted his postdoctoral training in Alan Jasanoff's laboratory at MIT before starting a laboratory at Helmholtz Center Munich (IBMI/IDG) and joining the medical faculty of Technische Universitaet Muenchen. The Westmeyer laboratory is developing next-generation molecular imaging and control technology to enable closed-loop cell circuit control with spatiotemporal precision and deep-tissue penetration for neuroscientific applications.

Arne Erdmann: Biography is not available. 